\title{
Rabi oscillations in semiconductor superlattices
}

\author{
Enrique Diez \\ GISC, Departamento de Matemáticas, Universidad Carlos III, E-28911 Leganés, Madrid, Spain
}

Rafael Gómez-Alcalá

Departamento de Tecnoloxía das Comunicacións, ETSET Universidade de Vigo, E-36200 Vigo, Spain

Francisco Domínguez-Adame

GISC, Departamento de Física de Materiales, Universidad Complutense, E-28040 Madrid, Spain

Angel Sánchez

GISC, Departamento de Matemáticas, Universidad Carlos III, E-28911 Leganés, Madrid, Spain

Gennady P. Berman

Theoretical Division and CNLS, Mailstop B213, Los Alamos National Laboratory, Los Alamos, New Mexico 87545

(Received 4 March 1998; revised manuscript received 2 April 1998)

\begin{abstract}
We investigate the dynamics of a semiconductor superlattice driven by an ac electric field by solving numerically the one-dimensional time-dependent Schrödinger equation including interface roughness effects. Rabi oscillations between minibands are clearly identified under resonant conditions, but we show that their lifetime is controlled by a characteristic time that depends on the superlattice deviation from perfection and is independent of the electric field. Experiments for the observation of the predicted effects are proposed. [S0163-1829(98)01327-7]
\end{abstract}

Coherent phenomena in semiconductor nanostructures are the basis for a new generation of ultrahigh-speed devices. Recently, it has become possible to experimentally investigate coherent effects in time-dependent transport in mesoscopic systems, ${ }^{1,2}$ opening the possibility to study qualitatively new effects that depend in a crucial way on the spatiotemporal coherence of the electronic states. Following these results, several works have been devoted to the analysis of the effects of time-dependent fields on the transport properties of resonant heterostructures. ${ }^{3-8}$

Within this context, in this work we present a full treatment of Rabi oscillations (RO's) between minibands of a semiconductor superlattice (SL). A tight-binding model of an ideal two-band system predicts the occurrence of Rabi oscillations. ${ }^{7}$ However, actual heterostructures present a certain degree of disorder that has been proven to be the responsibility of the observed fast dephasing of Bloch oscillations. ${ }^{9-11}$ Moreover, one expects that scattering effects due to disorder in imperfect SL's should be more important than in smaller devices (e.g., quantum wells and dots) because of their accumulated influence along the system. In addition, RO's could be even destroyed and turned into incoherent transitions between Bloch bands in the presence of disorder, as it has been suggested by Zhao et al. ${ }^{7}$ Clearly, this point is crucial from the experimental point of view if RO's are to be observed in actual SL's. The general motivation of our study is to point out the relevance of the unintentional disorder in coherent transport phenomena in SL's as a dephasing mechanism, in contrast to the general belief that due to the high quality of actual SL's this effect is negligible compared with other dephasing mechanisms.

The aim of this work is twofold. First, we present a realistic model, beyond the tight-binding approximation, con- taining all ingredients of actual SL's, namely, finite interband coupling, multiband scattering, and interface roughness. Second, and most important, we discuss the conditions under which RO's can be observed in an experiment of SL's driven by a strong laser field. One of our main findings is that RO's are extremely sensitive to small amounts of disorder, thus indicating that more elaborate models must take into account the imperfections of SL's.

The oscillations of a two-level system between the ground and excited states in the presence of a strong resonant driving field, often called transient nutation or Rabi oscillation, are discussed in textbooks ${ }^{12}$ as a topic of time-dependent perturbation theory. Consider a two-state system with ground-state energy $E_{0}$ and excited-state energy $E_{1}$ in the presence of a harmonic perturbation. If the frequency of the perturbation matches roughly the spacing between the two levels, the system undergoes oscillations with a frequency $\Omega_{R}$, which is much smaller than the excitation frequency $\omega_{\mathrm{ac}}$. This Rabi frequency depends on the mismatch $\delta \omega \equiv\left(E_{1}-E_{0}\right) / \hbar-\omega_{\mathrm{ac}}$ between the level spacing and the excitation frequency, and on the matrix element $F_{10}$ of the perturbation $\Omega_{R}=\left(\delta \omega^{2}\right.$ $\left.+\left|F_{10}\right|^{2} / \hbar^{2}\right)^{1 / 2}$. If we start with the system initially in the ground state, transitions between the ground and the excited state will occur with a period $T_{R}=2 \pi / \Omega_{R}$ as time evolves.

Here we answer the following question: Is this naive description valid for a real SL? Semiconductor superlattices present Bloch minibands with several states of each one; thus it is not clear whether they can be correctly described as a pure two-state system. We should also take into account the presence of imperfections introduced during growth processes. Interface roughness appearing during growth in $a c$ tual SL's depends critically on the growth conditions. ${ }^{13}$ For 
instance, protrusions of one semiconductor into the other cause in-plane disorder and break translational invariance parallel to the layers. To describe local excess or defect of monolayers, we allow the quantum well widths to fluctuate uniformly around the nominal values; this can be seen as substituting the nominal sharp width by an average along the parallel plane of the interface imperfections. Our approximation is valid whenever the mean free path of electrons is much smaller than the in-plane average size of protrusions, as electrons only see micro-quantum-wells with small areas and uniform thickness. ${ }^{13}$ Therefore, in the following we will take the width of the $n$th quantum well to be $a\left(1+W \epsilon_{n}\right)$, and the width of the $n$th barrier as $b\left(1+W \epsilon_{n}\right)$ where $W$ is a positive parameter measuring the maximum fluctuation, $\epsilon_{n}$ 's are distributed according to a uniform probability distribution, $\mathcal{P}\left(\epsilon_{n}\right)=1$ if $\left|\epsilon_{n}\right|<1 / 2$ and zero otherwise, $a$ is the nominal quantum well width, and $b$ is the nominal quantum barrier width.

For our present purposes, it is enough to focus on electron states close to the conduction-band edge and use the effective-mass approximation. The envelope function for the electron wave packet satisfies the following quantum evolution equation:

$$
\begin{aligned}
i \hbar \frac{\partial \Psi(x, t)}{\partial t}= & {\left[-\frac{\hbar^{2}}{2 m^{*}} \frac{d^{2}}{d x^{2}}+V_{\mathrm{SL}}(x)\right.} \\
& \left.-e F x \sin \left(\omega_{\mathrm{ac}} t\right)\right] \Psi(x, t),
\end{aligned}
$$

where $x$ is the coordinate in the growth direction, and $F$ and $\omega$ are the strength and the frequency of the ac field. The SL potential at flatband is $V_{\mathrm{SL}}(x)=\Delta E_{c}$ if $x$ lies inside the barriers and zero otherwise, $\Delta E_{c}$ being the conduction-band offset. We have considered a constant effective mass $m^{*}$ at the $\Gamma$ valley for simplicity. We use a uniformly spaced set of spatial mesh points much larger than the SL under consideration, and we transform the continuous boundary conditions, which read $\Psi(-\infty, t)=\Psi(\infty, t)=0$, to the corresponding discrete ones $\Psi_{-1}^{k}=\Psi_{N}^{k}=0$.

The band structure at flatband is computed by using a finite-element method. ${ }^{14}$ The eigenstate $j$ of the band $i$ with eigenenergy $E_{i}^{(j)}$ is denoted as $\psi_{i}^{(j)}(x)$. A good choice for the initial wave packet is provided by using a linear combination of the eigenstates belonging to the first miniband. For the sake of clarity we have selected as the initial wave packet $\Psi(x, 0)=\psi_{i}^{(j)}(x)$, although we have checked that this assumption can be dropped without changing our conclusions. The subsequent time evolution of the wave packet $\Psi(x, t)$ is calculated numerically by means of an implicit integration schema. ${ }^{14}$ In addition to $\Psi(x, t)$ we also compute the probability of finding an electron, initially in the state $\Psi(x, 0)$ $=\psi_{i}^{(j)}(x)$, in the state $\psi_{k}^{(j)}(x)$,

$$
P_{i k}^{(j)}(t)=\int_{-\infty}^{\infty} d x \Psi^{*}(x, t) \psi_{k}^{(j)}(x)
$$

We present here results for a SL with ten periods of 100- $\AA \mathrm{GaAs}$ and $50-\AA \mathrm{Ga}_{0}{ }_{7} \mathrm{Al}_{03} \mathrm{~A}$ with $\Delta E_{c}=250 \mathrm{meV}$ and $m^{*}=0.067 m, m$ being the free electron mass. We consider electric fields from 12.5 up to $100 \mathrm{kV} / \mathrm{cm}$. The fluctuation

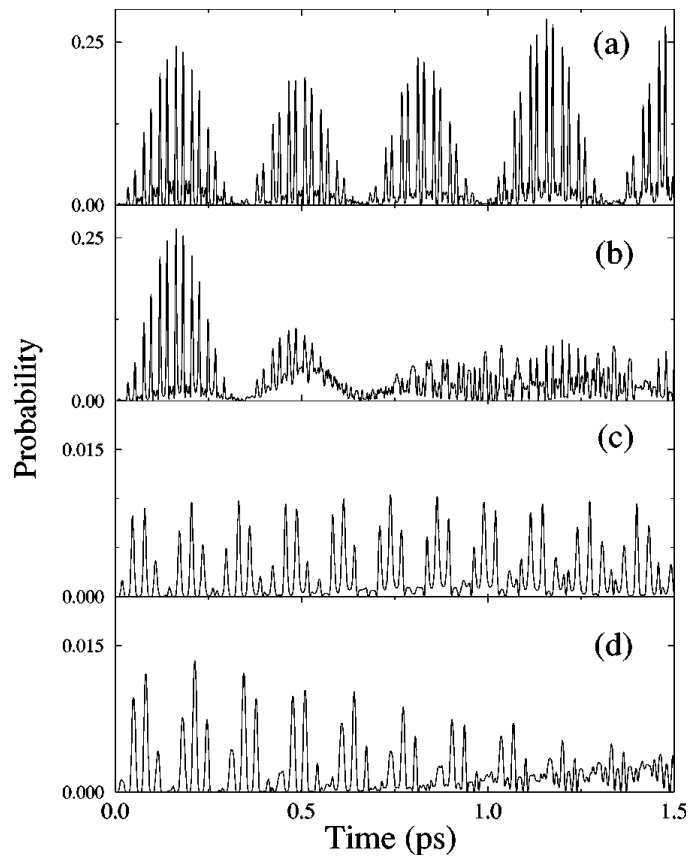

FIG. 1. The probability of finding an electron, initially situated in $\psi_{0}^{(5)}(x)$, in the state $\psi_{1}^{(5)}(x)$ as a function of time for $F$ $=50 \mathrm{kV} / \mathrm{cm}$, when the ac field is tuned to the resonant frequency $\omega_{\mathrm{ac}}=\omega_{\mathrm{res}}=150 \mathrm{THz}$ with (a) $W=0$ (perfect SL) and (b) $W=0.03$. Results for a perfect SL driven by an ac field out of the resonant regime (c) $\omega_{\mathrm{ac}}=100$ and (d) $200 \mathrm{THz}$ are also shown. Note the different vertical scales.

parameter runs from $W=0$ (perfect SL's) up to $W=0.03$ (less than $1 \mathrm{ml}$ ). Figure 1 displays $P_{01}^{(5)}(t)$ with $F$ $=50 \mathrm{kV} / \mathrm{cm}$, for different values of the external ac field frequency $\omega_{\mathrm{ac}}$. In this way, we are monitoring the transitions between the central state $(j=5)$ in the first miniband to the central state in the second miniband as a function of time. In Fig. 1(a) we observe the occurrence of very well-defined RO's when the ac field is tuned to the resonant frequency $\omega_{\text {res }}=\left(E_{1}^{(5)}-E_{0}^{(5)}\right) / \hbar=150 \mathrm{THz}$ in a perfect $\mathrm{SL}(W=0)$. Summing up the probabilities of the rest of the states in the second miniband, the probability of finding the electron in this band is very close to unity $(\sim 0.99)$. The frequency of the RO's, obtained performing the fast Fourier transform (FFT) of $P_{01}^{(5)}(t)$ is $\omega_{\mathrm{Rabi}}=19.18 \mathrm{THz}$.

Notice, however, that the perfect oscillatory pattern detected in perfect SL's is completely altered when we introduce a small amount of disorder. In Fig. 1(b) we consider a maximum excess or defect of monolayers less than $1 \mathrm{ml}$ ( $W=0.03$ ) whereas the rest of the parameters are the same. We observe that the perfect oscillatory pattern is destroyed after less than $1 \mathrm{ps}$. The disorder induces both a decrease of the amplitude of the oscillations and a progressive dephasing compared with the ideal perfect case in Fig. 1(a). This fact can be explained by the scattering of electrons with the random potential. Figures 1(c) and 1(d) show the behavior of $P_{01}^{(5)}(t)$ for a perfect SL but out of resonance with $\omega_{\mathrm{ac}}$ $=100$ and $200 \mathrm{THz}$, respectively. These frequencies try to pump the electron to energies situated in the gaps below and over the second miniband and then we detect negligible populations of the second miniband. Their FFT only shows the value of $\omega_{\mathrm{ac}}$ introduced by the external field. 


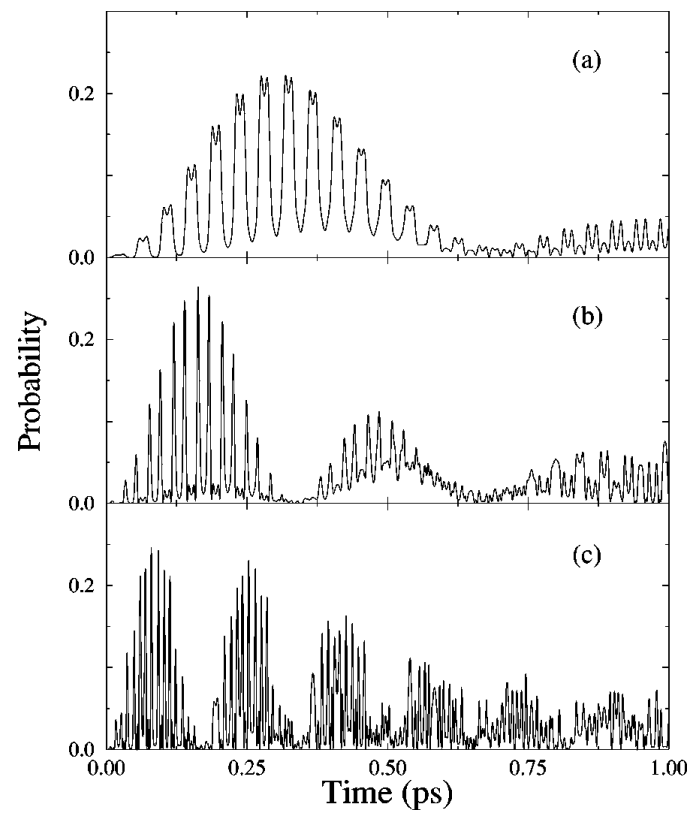

FIG. 2. The probability of finding the electron in the state $\psi_{1}^{(5)}(x)$ as a function of time for $W=0.03, \omega_{\mathrm{ac}}=\omega_{\mathrm{res}}=150 \mathrm{THz}$ and (a) $F=25$, (b) 50 , and (c) $100 \mathrm{kV} / \mathrm{cm}$.

From the above results we conjecture that there exists a characteristic scattering time $\tau_{\text {dis }}$ related to the amount of disorder in the sample, after which RO's are destroyed by disorder. Moreover, it is readily observed that $\tau_{\text {dis }}$ decreases upon increasing the degree of disorder. However, the above results have been obtained for a fixed value of the electric field. To check the validity of the introduced $\tau_{\text {dis }}$ we have studied $P_{01}^{(5)}(t)$ for different values of the ac field strength at the same amount of disorder of Fig. 1(b). Typical results are presented in Fig. 2 for $W=0.03$ and $F=25,50$, and 100 $\mathrm{kV} / \mathrm{cm}$. From this plot we can estimate that $\tau_{\mathrm{dis}} \simeq 0.75 \mathrm{ps}$ for all values of the electric field. Thus, this scattering time plays the same role as the scattering time arising from inelastic interactions, in the sense that the period of RO's must be kept smaller than $\tau_{\text {dis }}$ to be observed. The behavior of RO's is very similar to the case when disorder is absent for $t$ $<\tau_{\text {dis }}$. Moreover, performing a FFT of $P_{01}^{(5)}(t)$ for different values of the electric field we obtain $\omega_{\text {Rabi }}$ that shows a linear dependence with the field, expected from perturbation theory, with very few differences between the values of $\omega_{\text {Rabi }}$ in perfect or imperfect SL's for $t<\tau_{\text {dis }}$.

Upon activation by an infrared field, a particle in the first miniband can be activated to the second miniband where the probability of tunneling to the free region is several orders of magnitude higher. Figure 3 shows the integrated probability density in the right part of the SL, defined as

$$
P_{T}(t)=\int_{x_{r}}^{\infty}|\Psi(x, t)|^{2} d x
$$

$x_{r}$ being the coordinate of the right edge of the SL for (a) $W=0$ and (b) 0.03 . Solid lines are the results in the resonant regime $\omega_{\mathrm{ac}}=\omega_{\mathrm{res}}=150 \mathrm{THz}$, whereas dashed lines are results for $\omega_{\mathrm{ac}}=200 \mathrm{THz}$ (out of resonance). We can see that the tunneling probability is negligible when the ac field fre-

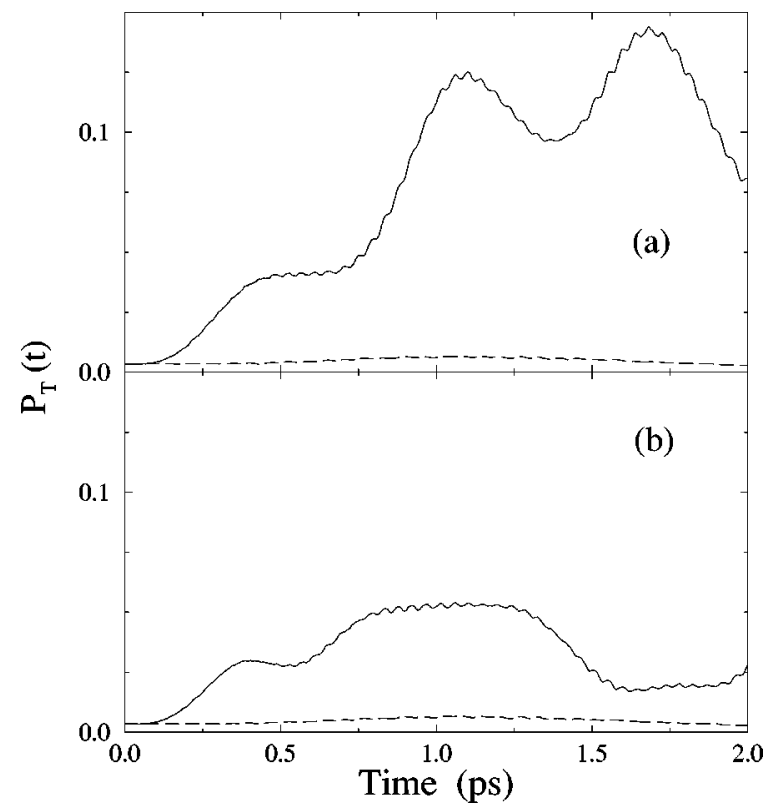

FIG. 3. $P_{T}(t)$ as function of time, for (a) $W=0$ and (b) 0.003 . Solid lines are the results in the resonant regime $\omega_{\mathrm{ac}}=\omega_{\mathrm{res}}=150 \mathrm{THz}$, whereas dashed lines are results for $\omega_{\mathrm{ac}}=200 \mathrm{THz}$ (out of resonance).

quency is not very close to the resonant one. When $\omega_{\mathrm{ac}}$ is close to the resonant frequency, the wave function is therefore emitted by bursts from the SL region every time a RO has been completed. We can see that this phenomenon is also observed for the imperfect SL for times smaller than $\tau_{\text {dis }}$.

Finally, some words about the experimental conditions to observe that the theoretical findings are in order. Nowadays a high-power terahertz ac field can be generated in a freeelectron laser. ${ }^{15}$ A straightforward way to observe RO in SL experimentally is to detect the radiation at the frequency $\omega_{\text {Rabi }}$ emitted by the oscillating dipole associated with the $\mathrm{RO}$ when the SL is driven by the ac field at the resonant frequency.

In summary, we have solved the dynamical problem of electrons moving in a semiconductor superlattice driven by an ac electric field. We found that the electron can perform RO's under resonant conditions but any degree of disorder due to imperfections during growth leads to the disappearance of RO's after a few oscillations: The higher the degree of disorder the faster the vanishing of RO's. Most importantly, we have been able to define a characteristic scattering time $\tau_{\text {dis }}$, independent of the electric field, after which RO's cannot be detected, this being a specific prediction of our model that can be checked in experiments. The existence of such a critical field is very important from the viewpoint of practical applications of our results. $\tau_{\text {dis }}$ is directly related to the degree of disorder present in the sample and decreases upon increasing the quality of the sample, i.e., it is an excellent parameter to assess the performance of epitaxial growth techniques. Finally, we have shown that electrons are emitted by bursts under resonant conditions, whereas the tunneling probability is vanishingly small out of resonance.

One of the main conclusions of this work is to underline the importance of disorder in the transport properties of SL's, in contrast to the general belief than the high quality of 
actual SL's make the disorder a second-order effect. It has to be kept in mind that an average disorder of less than $1 \mathrm{ml}$ is currently unavoidable. We note, however, that we have restricted the discussion to the single-electron case. The electron-electron interactions and other dephasing processes such as impurity or phonon scattering have been ignored and, in principle, could affect the predicted phenomena. Therefore, more theoretical work is needed to investigate the role of imperfections and many-body interactions on the coupling between the induced coherence and excitation. Work along these lines is currently in progress.

E.D. thanks Antti-Pekka Jauho for illuminating conversations and his warm hospitality at Mikroelektronik Centret where this paper was written in part. Work at Leganés and Madrid was supported by CICYT (Spain) under Projects Nos. MAT95-0325 and PB96-0199. E.D. gratefully acknowledges partial support from Fundación Universidad Carlos III de Madrid. G.P.B. gratefully acknowledges partial support by the Defense Advanced Research Projects Agency.
${ }^{1}$ J. B. Pieper and J. C. Price, Phys. Rev. Lett. 72, 3586 (1994).

${ }^{2}$ S. T. Cundiff, A. Knorr, J. Feldmann, S. W. Koch, E. O. Göbel, and H. Nickel, Phys. Rev. Lett. 73, 1178 (1994).

${ }^{3}$ T. Martin and G. P. Berman, Phys. Lett. A 196, 65 (1994).

${ }^{4}$ D. W. Hone and X.-G. Zhao, Phys. Rev. B 53, 4834 (1996).

${ }^{5}$ C. A. Stafford and N. S. Wingreen, Phys. Rev. Lett. 76, 1916 (1996).

${ }^{6}$ A.-P. Jauho and K. Johnsen, Phys. Rev. Lett. 76, 4576 (1996).

${ }^{7}$ X.-G. Zhao, G. A. Georgakis, and Q. Niu, Phys. Rev. B 54, R5235 (1996).

${ }^{8}$ A. Wacker, A. P. Jauho, S. Zeuner, and S. J. Allen, Phys. Rev. B 56, 13268 (1998).
${ }^{9}$ E. Diez, A. Sánchez, F. Domínguez-Adame, and G. P. Berman, Phys. Rev. B 54, 14550 (1996).

${ }^{10}$ J. P. Reynolds and M. Luban, Phys. Rev. B 54, R14 301 (1996).

${ }^{11}$ E. Diez, F. Domínguez-Adame, and A. Sánchez, Microelectron. Eng. 43, 103 (1998).

${ }^{12}$ C. Cohen-Tannoudji, B. Diu, and F. Laloë, Quantum Mechanics (Wiley, New York, 1977).

${ }^{13}$ K. A. Mäder, L.-W. Wang, and A. Zunger, J. Appl. Phys. 78, 6639 (1995)

${ }^{14}$ Details of the calculation will be given elsewhere.

${ }^{15}$ H. Drexler, J. S. Scott, S. J. Allen, K. L. Campman, and A. C. Gossard, Appl. Phys. Lett. 67, 2816 (1995). 